\title{
PROFUNDIDADE DE DEPOSIÇÃO DE SEMENTE DE MILHO NA REGIÃO DOS CAMPOS GERAIS, PARANÁ
}

\section{PEDRO H. WEIRICH NETO ${ }^{1}$, ADRIANA SCHIMANDEIRO ${ }^{2}$, LEANDRO M. GIMENEZ ${ }^{3}$, MARCELO J. COLET ${ }^{4}$, PAULO W. GARBUIO ${ }^{5}$}

\begin{abstract}
RESUMO: O sistema plantio direto encontra-se hoje plenamente incorporado à região dos Campos Gerais no Paraná. A acurácia e a precisão no processo de semeadura são itens de grande importância para que tal sistema alcance sucesso. Sendo assim, foram selecionadas 50 propriedades e, em 38, determinou-se a profundidade de deposição da semente de milho. A área experimental era formada por quatro ou cinco linhas de semeadura, onde se avaliaram dez plântulas por linha. A profundidade ficou em torno de $46 \mathrm{~mm}$, observando-se diferenças significativas entre as linhas, em 21 áreas. O coeficiente de variação médio ficou em torno de $20 \%$, limite estatístico entre médio e alto. Conforme análise de outros parâmetros, tais coeficientes podem representar diferentes tipos de erro no processo. Pode-se considerar o processo de semeadura da região como eficiente quando considerada a média da profundidade. Entretanto, a análise da variabilidade demonstra necessidade de ações junto a fatores antrópicos e de máquina.
\end{abstract}

PALAVRAS-CHAVE: plantio direto, processo de semeadura, mecanização agrícola.

\section{PLACEMENT DEPTH OF SEED CORN IN THE REGION OF CAMPOS GERAIS, PARANÁ, BRAZIL}

\begin{abstract}
No Tillage system is fully incorporated to farming in the region of Campos Gerais, state of Paraná. Accuracy and precision in the planting process are items of great importance for the success of this system. In order to evaluate the planting process, thirty eight farms were selected as sites for analysis of the placement depth of seeds. The research area was 4 or 5 planting rows, evaluating 10 plantlets per row. The average seed depth was around $46 \mathrm{~mm}$, and significant differences between rows were observed in 21 areas. The average coefficient of variation was around $20 \%$, the statistical limit between medium and high. Analyses of other parameters show that those coefficients may represent different errors in the process. The planting process in Campos Gerais can be considered efficient regarding to the average seed depth. However, the analysis of variability implies de need of actions concerning to anthropic and machinery factors.
\end{abstract}

KEYWORDS: no tillage, planting process, agricultural mechanization.

\section{INTRODUÇÃO}

A região dos Campos Gerais, no Estado do Paraná, possui características próprias dentro da agricultura brasileira. Pioneira no sistema plantio direto, apresenta alta tecnificação e rendimentos agrícolas elevados. A SEAB (2003) relata rendimento médio de milho para a referida região de $6,8 \mathrm{t} \mathrm{ha}^{-1}$. Com o objetivo de obter altos rendimentos, o processo produtivo deve respeitar algumas variáveis, desde a implantação da cultura, passando pelo processo de semeadura, propriedades do solo, condução fitotécnica e fitopatológica, até a colheita. Nessa identificação, o processo de

\footnotetext{
${ }^{1}$ Eng ${ }^{0}$ Agrícola, Prof. Doutor, Laboratório de Mecanização Agrícola (Lama), Universidade Estadual de Ponta Grossa (UEPG), Fone: (0xx42)3220-3092, lama1@uepg.br

${ }^{2}$ Eng $^{\mathrm{o}}$ Agrônomo, M. Sc., Programa de Pós-Graduação em Manejo Sustentável de Recursos, Universidade Tecnológica de Munique (TUM), Freising, Alemanha.

${ }^{3}$ Engo Agrônomo, M. Sc., Fundação ABC para Assistência e Divulgação Técnica Agropecuária, Castro - PR.

${ }^{4}$ Eng ${ }^{\mathrm{o}}$ Agrônomo, M. Sc., Programa de Pós-graduação em Engenharia Agrícola, Universidade Estadual de Campinas (UNICAMP), Campinas, SP.

${ }^{5}$ Eng ${ }^{\mathrm{o}}$ Agrônomo, Programa de Pós-Graduação em Agronomia, UEPG, Ponta Grossa - PR.

Recebido pelo Conselho Editorial em: 5-1-2007
}

Aprovado pelo Conselho Editorial em: 25-10-2007 
semeadura, representado pela distribuição e profundidade de deposição de sementes de milho, temse mostrado influente.

O objetivo deste trabalho foi quantificar a profundidade de deposição de sementes de milho em áreas comerciais sob sistema plantio direto, na região dos Campos Gerais, Paraná.

\section{DESCRIÇÃO DO ASSUNTO}

Devido à presença da cobertura vegetal e ao adensamento natural das partículas do solo, o processo de semeadura foi a operação que sofreu as maiores transformações no sistema plantio direto. Dentro do processo de semeadura, LIU et al. (2004) relataram haver maior correlação do rendimento do milho com a variabilidade de emergência do que com a distribuição de plantas.

Estudando a emergência de milho, GUPTA et al. (1988) observaram que, em temperaturas favoráveis, existe correlação linear positiva entre a profundidade de deposição da semente e o tempo necessário para a emergência das plântulas. PRADO et al. (2001) não encontraram diferenças para a velocidade de emergência em diferentes profundidades de semeadura, em experimento com suplementação hídrica. YORINORI et al. (1996) descreveram, sem relato do conteúdo de água do solo, proporcionalidade inversa entre profundidade e velocidade de emergência de milho-pipoca.

Alguns autores citam profundidades consideradas adequadas: FANCELLI \& DOURADO NETO (2000) descrevem que essa seria entre 3 e $5 \mathrm{~cm}$ para solos argilosos e 4 e $6 \mathrm{~cm}$ para solos arenosos. WEIRICH NETO (2004), estudando 60 pontos em uma lavoura comercial, relatou $3,9 \mathrm{~cm}$ como profundidade ideal de deposição da semente, onde sementes depositadas abaixo ou acima dessa profundidade necessitaram de tempo maior para emergir.

Embora a profundidade de semeadura seja importante, MANTOVANI \& BERTAUX (1990) relatam dificuldade no controle da mesma pelos mecanismos disponíveis nas semeadoras. Considerando o discutido, SATTLER (1992) idealizou e testou dispositivo para controle da profundidade, alcançando controle eficiente da profundidade regulada; porém, até o presente, o mesmo não se encontra disponível comercialmente, lembrando que, no sistema plantio direto, existem agravantes, como a presença de resíduos, irregularidade da superfície e grande variabilidade da resistência do solo.

As áreas avaliadas pertencem a produtores filiados às Cooperativas Capal, de Arapoti; Batavo, de Carambeí, e Castrolanda, de Castro. A escolha das áreas foi a partir de imagem de satélite, na qual se identificaram as propriedades agendadas para receberem os híbridos de milho TORK $^{\circledR}$ e DKB-214 ${ }^{\circledR}$, na safra agrícola 2001/2002, apontados pelo setor de compras das cooperativas como os mais cultivados. Para amostragem espacial homogênea, sobrepôs-se uma malha de $5 \mathrm{~km}$ à imagem, selecionando-se uma das propriedades identificadas em cada quadro.

Foram selecionadas 38 propriedades para avaliar a profundidade de semeadura. Em cada propriedade, delimitou-se uma área experimental formada por quatro ou cinco linhas de semeadura, conforme a semeadora utilizada, sendo $60,5 \%$ dessas com sistema sulcador de fertilizante tipo haste, $81,5 \%$ com sistema dosador de sementes tipo disco perfurado horizontal e com grande variedade de discos de corte de palha. Para avaliar a profundidade de deposição, cortou-se a plântula rente ao solo, dez dias após o início da emergência, retirando-se cuidadosamente a raiz e medindo-se a distância do corte até a semente, com dez repetições por linha. Os dados foram analisados por estatística descritiva e análise de variância. Nos casos em que se constataram diferenças significativas entre as linhas, utilizou-se do teste de Tukey, a 5\% de probabilidade de erro.

Verificaram-se, conforme Tabela 1, valores extremos para o coeficiente de variação (C.V.), como nas áreas 4; 26; 30 e 34. Em análise mais detalhada, observa-se que, na área 30, a variabilidade é grande, indicando distribuição platicúrtica. Nesse pôde-se identificar visualmente a quase inexistência de material de cobertura e solo pouco estruturado e/ou conteúdo de água baixo 
no momento da semeadura, ocorrendo no sulco de semeadura grandes torrões. Já no caso da área 4, a distribuição de freqüência apresentou valores discrepantes (outliers). Nesse caso, as condições de conservação da semeadora não eram as ideais.

TABELA 1. Estatística descritiva dos dados de profundidade ( $\mathrm{mm})$ de deposição de semente.

\begin{tabular}{|c|c|c|c|c|c|c|}
\hline \multirow{2}{*}{ Área } & \multirow{2}{*}{$\begin{array}{l}\text { Número de } \\
\text { Valores }\end{array}$} & \multicolumn{2}{|c|}{ Valor Mínimo Valor Máximo } & Média & Desvio-Padrão & \multirow{2}{*}{ C.V. $(\%)$} \\
\hline & & \multicolumn{4}{|c|}{$\mathrm{mm}$} & \\
\hline 1 & 40 & 36 & 80 & 60,3 & 10,0 & 16,6 \\
\hline 2 & 32 & 36 & 74 & 56,4 & 9,6 & 17,0 \\
\hline 3 & 32 & 25 & 84 & 55,8 & 11,7 & 21,0 \\
\hline 4 & 40 & 5 & 69 & 37,3 & 16,6 & 44,6 \\
\hline 5 & 40 & 12 & 59 & 37,1 & 9,2 & 24,9 \\
\hline 6 & 40 & 20 & 61 & 43,8 & 11,3 & 25,9 \\
\hline 7 & 40 & 11 & 56 & 40,8 & 9,4 & 23,1 \\
\hline 8 & 50 & 26 & 63 & 41,0 & 9,0 & 21,9 \\
\hline 9 & 40 & 34 & 79 & 58,3 & 8,6 & 14,8 \\
\hline 10 & 32 & 25 & 49 & 39,4 & 6,4 & 16,2 \\
\hline 11 & 32 & 15 & 61 & 46,1 & 10,9 & 23,7 \\
\hline 12 & 32 & 35 & 76 & 56,5 & 10,3 & 18,2 \\
\hline 13 & 40 & 35 & 75 & 57,6 & 10,2 & 17,7 \\
\hline 14 & 32 & 20 & 59 & 36,8 & 9,2 & 24,9 \\
\hline 15 & 40 & 25 & 51 & 39,1 & 6,0 & 15,2 \\
\hline 16 & 40 & 34 & 62 & 49,8 & 7,4 & 14,9 \\
\hline 17 & 40 & 24 & 61 & 43,1 & 8,4 & 19,6 \\
\hline 18 & 32 & 25 & 65 & 42,0 & 10,5 & 25,1 \\
\hline 19 & 40 & 21 & 56 & 36,2 & 8,9 & 24,5 \\
\hline 20 & 33 & 11 & 41 & 27,0 & 6,8 & 25,2 \\
\hline 21 & 32 & 43 & 64 & 55,0 & 5,8 & 10,5 \\
\hline 22 & 32 & 16 & 46 & 30,7 & 8,8 & 28,8 \\
\hline 23 & 40 & 33 & 77 & 52,7 & 12,4 & 23,5 \\
\hline 24 & 32 & 30 & 70 & 51,6 & 9,5 & 18,4 \\
\hline 25 & 32 & 35 & 55 & 44,9 & 5,5 & 12,1 \\
\hline 26 & 40 & 20 & 75 & 47,9 & 14,2 & 29,6 \\
\hline 27 & 50 & 40 & 75 & 59,2 & 7,4 & 12,6 \\
\hline 28 & 40 & 33 & 66 & 51,6 & 9,8 & 18,9 \\
\hline 29 & 32 & 25 & 80 & 57,6 & 11,8 & 20,5 \\
\hline 30 & 50 & 16 & 68 & 39,1 & 12,4 & 31,6 \\
\hline 31 & 50 & 23 & 99 & 61,6 & 15,4 & 25,0 \\
\hline 32 & 40 & 48 & 74 & 60,1 & 7,9 & 13,1 \\
\hline 33 & 32 & 25 & 55 & 41,2 & 9,0 & 21,8 \\
\hline 34 & 32 & 11 & 34 & 21,3 & 6,7 & 31,6 \\
\hline 35 & 32 & 33 & 65 & 49,2 & 9,4 & 19,2 \\
\hline 36 & 32 & 23 & 51 & 37,9 & 7,7 & 20,3 \\
\hline 37 & 32 & 38 & 64 & 51,5 & 7,2 & 13,9 \\
\hline 38 & 32 & 25 & 62 & 40,3 & 8,6 & 21,3 \\
\hline Média & 37 & 26,1 & 64,8 & 46,2 & 9,5 & 21,3 \\
\hline
\end{tabular}

O C.V. médio das áreas estudadas ficou próximo de $20 \%$, valor classificado estatisticamente como alto (GOMES, 1990). Porém, devido à interação da regulagem da máquina (ação antrópica) e ao meio (cobertura e solo), o fenômeno natural é transformado, o que pode caracterizar valores de C.V. ao redor de $20 \%$ como normais. Tal afirmação é confirmada por CASÃO JÚNIOR et al. (1998) e CASÃO JÚNIOR et al. (2000), que encontraram C.V. de 22,9\% a 41,6\% e de 26,3\% a 
$32,7 \%$ nas linhas, para a profundidade de deposição de semente em sistema plantio direto. Analisando duas linhas de uma semeadora, WEIRICH NETO (2004) relatou valores de 21,8\% e $28,8 \%$. BATEMAN (1972) encontrou desvios-padrão maiores quanto maior a profundidade de deposição.

Contudo, observa-se C.V. de 10,5 e 12,1\%. Se alguns produtores alcançam esses valores, presume-se que tais valores sejam possíveis. Nesse caso, podem-se adotar esses valores como meta para produtores, padrão de avaliação de processo, bem como objetivo em ações de treinamentos.

Das 38 áreas avaliadas, 21 apresentaram diferença significativa entre as médias de profundidade de deposição de sementes das linhas de semeadura (Tabela 2). Essa observação indica a influência de variáveis antrópicas (regulagem da semeadora), bem como de variáveis de projeto da semeadora (como molas com deformações diferentes) já relatadas por MORISON JÚNIOR \& GERIC (1985).

TABELA 2. Comparação de médias das profundidades $(\mathrm{mm})$ de deposição de sementes encontradas por linha de semeadura.

\begin{tabular}{cllllc}
\hline Área & Linha 1 & Linha 2 & Linha 3 & Linha 4 & Linha 5 \\
\hline 1 & $70,4 \mathrm{~b}$ & $56,3 \mathrm{a}$ & $63,0 \mathrm{ab}$ & $51,1 \mathrm{a}$ & - \\
4 & $38,2 \mathrm{~b}$ & $19,6 \mathrm{a}$ & $33,0 \mathrm{~b}$ & $58,5 \mathrm{c}$ & - \\
6 & $37,4 \mathrm{a}$ & $53,2 \mathrm{~b}$ & $51,0 \mathrm{~b}$ & $33,5 \mathrm{a}$ & - \\
7 & $46,5 \mathrm{~b}$ & $35,5 \mathrm{a}$ & $37,0 \mathrm{ab}$ & $44,3 \mathrm{ab}$ & - \\
8 & $47,0 \mathrm{~b}$ & $40,1 \mathrm{ab}$ & $44,0 \mathrm{ab}$ & $36,8 \mathrm{a}$ & $36,8 \mathrm{a}$ \\
10 & $34,4 \mathrm{a}$ & $43,8 \mathrm{~b}$ & $35,9 \mathrm{a}$ & $43,8 \mathrm{~b}$ & - \\
12 & $64,5 \mathrm{~b}$ & $59,4 \mathrm{~b}$ & $53,0 \mathrm{a}$ & $49,0 \mathrm{a}$ & - \\
13 & $64,4 \mathrm{~b}$ & $48,4 \mathrm{a}$ & $56,0 \mathrm{ab}$ & $61,5 \mathrm{~b}$ & - \\
15 & $33,6 \mathrm{a}$ & $40,2 \mathrm{~b}$ & $42,5 \mathrm{~b}$ & $40,0 \mathrm{~b}$ & - \\
16 & $48,3 \mathrm{ab}$ & $53,7 \mathrm{~b}$ & $42,6 \mathrm{a}$ & $54,6 \mathrm{~b}$ & - \\
17 & $41,2 \mathrm{~b}$ & $50,6 \mathrm{c}$ & $33,1 \mathrm{a}$ & $47,3 \mathrm{c}$ & - \\
18 & $40,6 \mathrm{a}$ & $53,5 \mathrm{~b}$ & $36,6 \mathrm{a}$ & $37,1 \mathrm{a}$ & - \\
20 & $28,1 \mathrm{ab}$ & $30,2 \mathrm{~b}$ & $20,9 \mathrm{a}$ & $28,4 \mathrm{ab}$ & - \\
22 & $39,4 \mathrm{~b}$ & $32,5 \mathrm{ab}$ & $27,3 \mathrm{a}$ & $23,5 \mathrm{a}$ & - \\
27 & $57,1 \mathrm{a}$ & $54,7 \mathrm{a}$ & $59,8 \mathrm{ab}$ & $57,5 \mathrm{a}$ & $66,7 \mathrm{~b}$ \\
28 & $51,8 \mathrm{~b}$ & $53,4 \mathrm{bc}$ & $40,8 \mathrm{a}$ & $60,4 \mathrm{c}$ & - \\
29 & $62,5 \mathrm{~b}$ & $62,4 \mathrm{~b}$ & $57,9 \mathrm{ab}$ & $47,8 \mathrm{a}$ & - \\
30 & $29,7 \mathrm{a}$ & $54,5 \mathrm{c}$ & $43,9 \mathrm{~b}$ & $33,1 \mathrm{a}$ & $34,3 \mathrm{ab}$ \\
31 & $63,8 \mathrm{~b}$ & $44,6 \mathrm{a}$ & $77,9 \mathrm{c}$ & $62,8 \mathrm{~b}$ & $58,9 \mathrm{~b}$ \\
32 & $54,8 \mathrm{a}$ & $60,4 \mathrm{ab}$ & $59,9 \mathrm{ab}$ & $65,3 \mathrm{~b}$ & - \\
36 & $42,5 \mathrm{~b}$ & $41,6 \mathrm{~b}$ & $38,6 \mathrm{~b}$ & $28,9 \mathrm{a}$ & - \\
\hline
\end{tabular}

Valores seguidos de mesma letra na horizontal não diferem significativamente, conforme teste de Tukey, a 5\% de significância.

Quanto maior a profundidade de deposição, maior o consumo de energia na emergência, além de prejuízos causados por baixas temperaturas e baixos níveis de oxigênio; já quanto menor a profundidade, maior a suscetibilidade da semente a estresses hídricos. Plantas que emergem em diferentes velocidades, além do estresse inicial, poderão ter a taxa fotossintética reduzida devido ao sombreamento ou ter a polinização afetada. Em todos os casos, a heterogeneidade de emergência afeta o rendimento do milho (NAFZIGER et al., 1991).

Quanto às médias, foi possível constatar que, no geral, os valores foram próximos do recomendado para a cultura, que seria em torno de $50 \mathrm{~mm}$. Porém, isso não significa que as profundidades adotadas pelos produtores estivessem corretas ou incorretas, pois o valor ideal depende das condições edafoclimáticas de momento. 


\section{CONCLUSÕES}

Pelos valores médios de deposição de semente de milho, conclui-se que os produtores conhecem as recomendações agronômicas. Porém a variabilidade em torno dessa média demonstra algumas discrepâncias. O momento inadequado do processo de semeadura, a manutenção incorreta e o descaso nas regulagens entre as linhas da semeadora foram fatos observados. Ações referentes a treinamento devem ser implementadas.

\section{REFERÊNCIAS}

BATEMAN, H.P. Planter metering, soil and plant factors affecting corn ear populations. Transactions of the ASAE, St. Joseph, v.15, n.6, p.1013-20, 1972.

CASÃO JÚNIOR, R.; ARAÚJO, A.G. de; RALISCH, R.; SILVA, A. L. da; LADEIRA, A. S.; SILVA, J. C. da; MACHADO, P.; ROSETTO, R. Avaliação do desempenho da semeadoraadubadora Magnum 285-PD. Londrina: IAPAR, 1998. 47 p. (Circular 105).

CASÃO JUNIOR, R.; ARAÚJO, A. G. de; RALISCH, R.; MEDEIROS, G. B. de; MONICE, R,; SIQUEIRA, R.; SILVA, A. L. da; LADEIRA, A. S,; SILVA, J. C. da; MACHADO, P.; ROSETTO, R. Desempenho da semeadora-adubadora AS 13500-Vence Tudo em solos argilosos. Londrina: IAPAR, 2000. 46 p. (Circular 110).

FANCELLI, A.L.; DOURADO NETO, D. Fisiologia da produção e aspectos básicos de manejo para alto rendimento. In: SANDINI, I.E.; FANCELLI, A.L. Milho: estratégia de manejo para Região Sul. Guarapuava: Fundação Agrária de Pesquisa Agropecuária, 2000. 209 p.

GOMES, F. P. Curso de estatística experimental. 12. ed. São Paulo: Nobel, 1990. 467 p.

GUPTA, S.C.; SCHENEIDER, E.C.; SWAN, J.B. Planting depth and tillage interactions on corn emergence. Soil. Science Society of America Journal, Madison, v.52, n.4, p.1122-27, 1988.

LIU, W.; TOLLENAR, M.; STEWART, G.; DEEN, W. Response of corn grain yield to spatial and temporal variability in emergence. Crop Science, Madison, v.44, n.3, p.847-54, 2004.

MANTOVANI, E.C.; BERTAUX, S. Avaliação do desempenho de semeadoras-adubadoras de milho (Zea mays L.) no campo. Sete Lagoas: Embrapa/CNPMS/ABIMAQ-SINDIMAQ, 1990. 49p.

MORISON JÚNIOR, J.E.; GERIC, T.J. Planter depth control: II - Empirical testing and plant responses. Transactions of the ASAE, St. Joseph, v.28, n.6, p.1744-8, 1985.

NAFZIGER, E.D.; CARTER, P.R.; GRAHAM, E.E. Response of corn uneven emergence. Crop Science, Madison, v.31, n.3, p.811-15, 1991.

PRADO, R.M.; TORRES, J.L.; ROQUE, C.G.; COAN, O. Semente de milho sob compressão do solo e profundidades de semeadura: Influência no índice de velocidade de emergência. Scientia Agrária, Curitiba, v.2, n.1, p.45-9, 2001.

SEAB. SECRETARIA DE AGRICULTURA E ABASTECIMENTO DO PARANÁ. Comparativo de área, produção e produtividade - 00/01-01/02-02/03. Disponível em:

http://www.pr.gov.br/celepar/seab/deral. Acesso em: 25 maio 2003.

SATTLER, A. Controle automático de profundidade de semeadura. 1992. 86 f. Dissertação (Mestrado em Máquinas Agrícolas) - Faculdade de Engenharia Agrícola, Universidade Estadual de Campinas, Campinas, 1992.

YORINORI, N.A.; SADA, S.Y.; PISAIA, A. Efeito da profundidade de semeadura e do envelhecimento precoce de sementes de milho-pipoca (Zea mays L.) sobre a emergência e vigor de plantas. Agrárias, Curitiba, v.16, n.2, p.173-8, 1996.

WEIRICH NETO, P.H. Importância de Atributos Agronômicos para qualificação de semeadura do milho (Zea mays L.) no sistema Plantio Direto na Região dos Campos Gerais - PR. 2004. $147 \mathrm{f}$. Tese (Doutorado em Água e Solo) - Faculdade de Engenharia Agrícola, Universidade Estadual de Campinas, Campinas, 2004. 